\title{
Analyse radioisotopique par spectrométrie gamma haute résolution d'échantillons de sol prélevés le long du golfe de Gabès au voisinage d'une usine de phosphates
}

\author{
A. BEN FREDJ ${ }^{\prime}$, M. BARRAK ${ }^{1}$, L. GHEDIRA ${ }^{1}$, M. CHELBI $^{1}$, \\ H. BEN ABDELWAHED ${ }^{2}$, N. RGUIGUI ${ }^{2}$
}

(Manuscrit reçu le 16 février 2002, accepté le 8 octobre 2002)

RÉSUMÉ Dans le cadre d'une étude concernant l'impact sur l'environnement des usines de phosphates implantées dans la région du golfe de Gabès dans le sud tunisien, une série de mesures préliminaires a été récemment conduite. Douze échantillons de sol ont été prélevés le long du golf de Gabès au voisinage d'une usine de traitement des minerais de phosphates. L'analyse de ces échantillons de sol par spectrométrie gamma haute résolution a révélé la présence d'une activité gamma de plus de $1700 \mathrm{~Bq} / \mathrm{kg}$ due à la présence des radio-isotopes : ${ }^{212} \mathrm{~Pb},{ }^{226} \mathrm{Ra},{ }^{214} \mathrm{~Pb},{ }^{214} \mathrm{Bi}$, et ${ }^{235} \mathrm{U}$. Cette activité reste pratiquement constante même à une distance de plus d'un kilomètre du site industriel que ce soit dans te milieu terrestre que dans le milieu marin.

ABSTRACT High-resolution gamma spectrometry analysis of soil samples collected in the vicinity of phosphate plant in the region of Gabes in South Tunisia.

A set of preliminary measurements have been conducted in order to study the impact on the environment of the phosphates plants located in the golf of Gabes in south Tunisia. Twelve samples of soil have been collected in the vicinity of such a plant. The analyses of these samples have been achieved by high-resolution gamma spectrometry technique revealing a gamma activity of $1700 \mathrm{~Bq} / \mathrm{kg}$ due to the presence of the following radioisotopes: ${ }^{212} \mathrm{~Pb},{ }^{226} \mathrm{Ra},{ }^{214} \mathrm{~Pb},{ }^{214} \mathrm{Bi}$, and ${ }^{235} \mathrm{U}$. This activity remains practically constant even more than one kilometer far from the plant.

\section{Introduction}

L'exploitation des minerais de phosphate, en vue de la production d'acide phosphorique et d'engrais, donne lieu à une très grande quantité de résidus de phosphogypses. Ces déchets sont souvent stockés à l'air libre non loin de l'usine. Quant aux déchets liquides (boues), ils sont fréquemment déversés dans la mer.

\footnotetext{
1 Faculté des Sciences de Monastir, Département de Physique, 5000 Monastir, Tunisie.

2 Centre National des Sciences et Technologies Nucléaires, Sidi Thabet. Tunis, Tunisic
} 
Le but de ce travail est l'évaluation de l'impact radiologique concernant à la fois le milieu terrestre et marin. Ils sont à la fois de nature chimique : pollution marine, et radiologique : émanation de radon dans l'air (UNSCEAR, 1993), inhalation de poussières et migration des déchets par ruissellement...

Dans cette étude, nous nous sommes limités à l'impact radiologique sur l'environnement d'une usine de transformation des minerais de phosphates située non loin du bord de mer dans la région du golf de Gabès (Skhira). À cette fin, nous avons prélevé douze échantillons de sol terrestre et marin sur une distance de plus d'un kilomètre en aval du site industriel.

Pour l'analyse radiologique des échantillons collectés nous avons adopté la technique de spectrométrie gamma haute résolution via l'utilisation d'un détecteur germanium de haute pureté dopé au lithium (HPGeLi) et refroidi à l'azote liquide.

\section{Prélèvement des échantillons}

Douze échantillons de sol, ayant chacun une masse de $1,5 \mathrm{~kg}$, ont été prélevés en surface sur une profondeur de cinq centimètres en aval du site industriel. Ces échantillons ont été regroupés en fonction de deux paramètres $X$ et $Y$. Le premier étant l'éloignement par rapport à la bouche de déversement des boues, le second la distance par rapport à la côte.

Quatre lots : A, B, C, D, comprenant chacun trois échantillons ont ainsi été répertoriés. Les trois échantillons du lot $\mathrm{A}$ ont été prélevés prés de la bouche d'éjection des boues, ceux du lot B à 300 mètres. Les lots C et D ont été prélevés, successivement, à 800 et 1300 mètres. Les échantillons d'indice 1 ont été prélevés dans le sol sous-marin à $2 \mathrm{~m}$ du bord de 1'eau, ceux d'indices 2 et 3 ont été prélevés, successivement, à 2 et 7 mètres de la côte (Fig. 1).

\section{Préparation des échantillons}

Les échantillons prélevés ont été séchés dans une étuve à une température de $60{ }^{\circ} \mathrm{C}$ pendant une durée variant en fonction de son degré d'humidité entre 10 et 20 heures puis broyés en fines particules. Des boites en plastique de $600 \mathrm{~cm}^{3}$ de volume (Marinelli Beacker) ont été partiellement remplies afin de laisser de l'espace pour des dégagements gazeux (radon). La masse d'échantillon de sol finalement utilisée est comprise entre 500 et 700 grammes. Toutes les boîtes ont été par la suite scellées afin d'empêcher tout échappement de radon. Les boîtes ont été stockées pendant une durée minimale de vingt jours dans le but d'atteindre l'équilibre radioactif. 


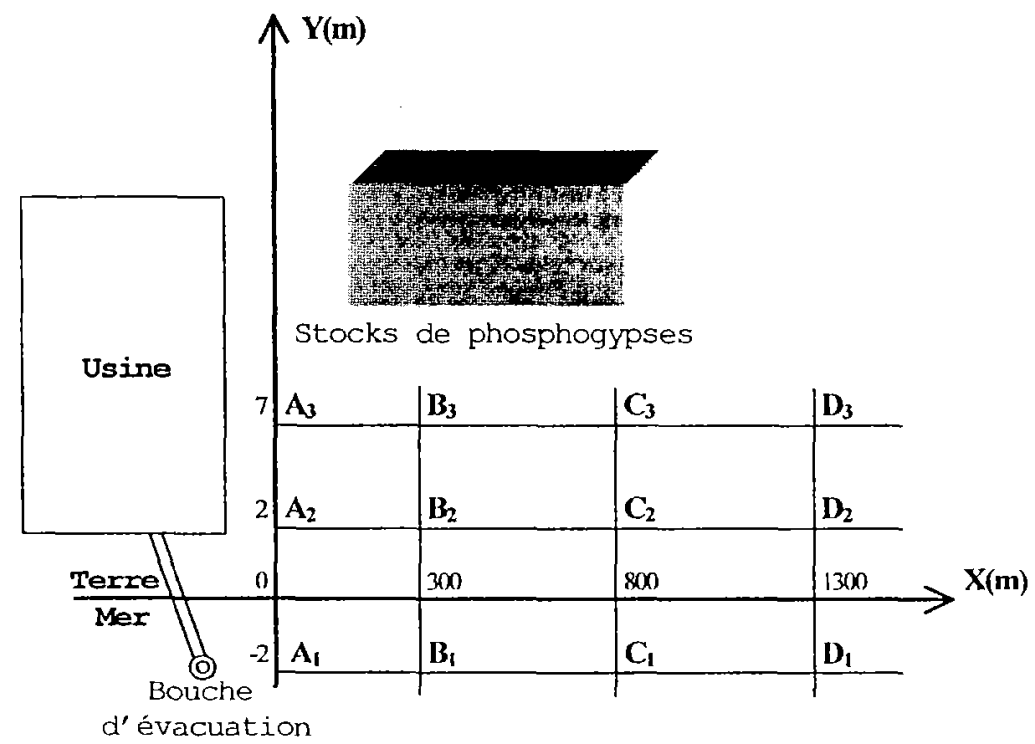

Figure 1 - Emplacement des échantillons par rapport à l'usine et au bord de l'eau.

Samples localization relative to the plant and the sea shore.

\section{Dispositif expérimental}

La chaîne de spectrométrie gamma utilisée comprend un détecteur germanium de haute pureté dopé au lithium (HPGeLi) et refroidi à l'azote liquide (Modèle GC 1520 Canberra), une alimentation haute tension (Modèle 3106D Canberra) réglée à une tension de $3500 \mathrm{~V}$, un préamplificateur (Modèle 2002 Canberra) placé au plus près du détecteur, un amplificateur (Modèle 2022 Canberra) réglé à un gain de 1000 et un temps de mise en forme (Shaping Time) de $2 \mu$ s, un analyseur multicanaux (Carte Accuspec A Canberra) comprenant 8192 canaux et fonctionnant à $100 \mathrm{MHz}$.

Le traitement des spectres d'amplitudes a été effectué grâce à un logiciel fourni par le fabricant (Logiciel Génie 2000).

\section{Mesures préliminaires}

\section{1. Étalonnage en énergie}

Sept sources étalons: ${ }^{241} \mathrm{Am},{ }^{109} \mathrm{Cd},{ }^{57} \mathrm{Co},{ }^{226} \mathrm{Ra},{ }^{137} \mathrm{Cs},{ }^{54} \mathrm{Mn}$ et ${ }^{60} \mathrm{Co}$, constituées de poudres conditionnées dans des boîtes Marinelli Baecker, ont été 


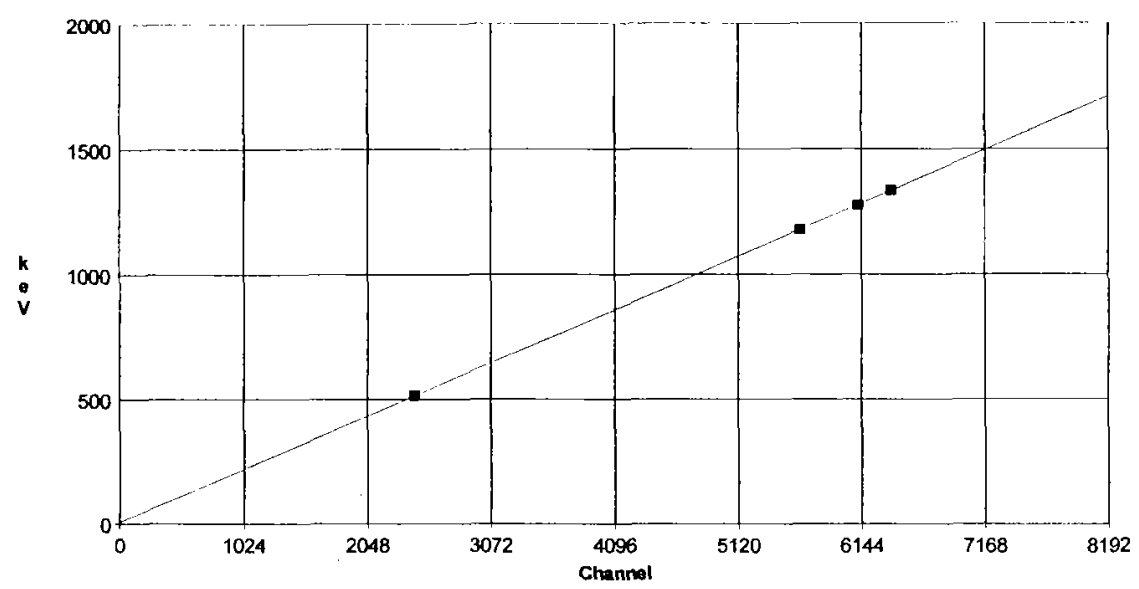

Figure 2-Etalonnage en énergie.

Energy calibration.

utilisées à la fois pour l'étalonnage en énergie et pour la mesure de l'efficacité de détection.

Le logiciel fourni par le fabricant permet la détermination automatique par la méthode des moindres carrés de la courbe d'étalonnage. Dans la plage d'énergie exploitée $(60$ à $1330 \mathrm{keV})$ la réponse du détecteur est parfaitement linéaire (Fig. 2).

\subsection{Efficacité de détection}

La plupart des photons interceptant le cristal de germanium le traversent sans laisser de trace (échappements) ou une trace inexploitable (effet Compton). Les seuls photons pris en compte sont ceux dont l'énergie est complètement transférée aux électrons atomiques sous forme d'énergie cinétique et totalement déposée dans le cristal (pic d'absorption totale). L'activité mesurée ne représente souvent que quelques pour-cent de l'activité réelle de l'échantillon à étudier.

Sachant que les activités des sources étalons fournies par le fabricant sont connues ainsi que la date de la mesure de ces activités, le rapport de l'activité mesurée avec notre chaîne sur celle calculée, donne l'efficacité globale de détection du système (Fig. 3). 


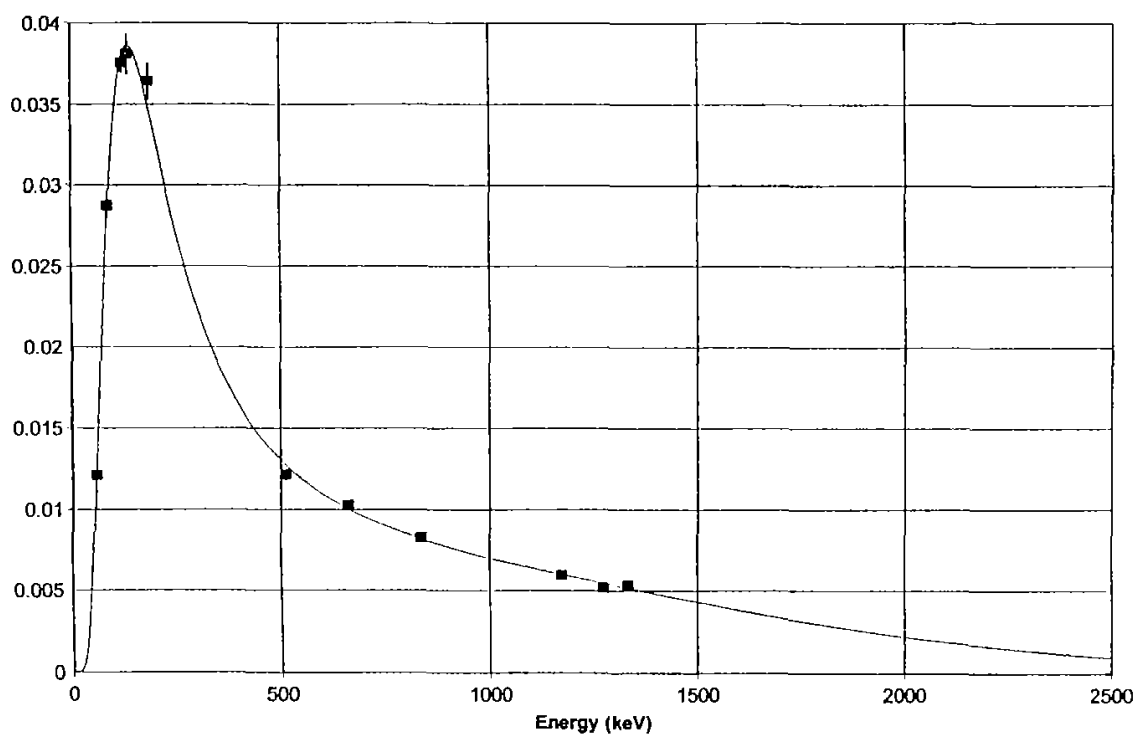

Figure 3 - Étalonnage en efficacité de détection.

Detection efficiency calibration.

\subsection{Bruit de fond}

Afin de soustraire les contributions parasites dues, d'une part, à la boite en plastique, d'autre part, au bruit de fond ambiant, un comptage à vide a été réalisé pendant une durée de temps égale à celle de l'acquisition des spectres.

La contribution du bruit de fond pour l'ensemble des radio-isotopes présents dans les échantillons est inférieure à $10 \%$ sauf pour le radio-isotope ${ }^{40} \mathrm{~K}$, dont la contribution au bruit de fond est prépondérante.

\section{Les mesures}

Pour chacun des échantillons, la durée d'acquisition a été fixée à $50000 \mathrm{~s}$ soit un peu moins de 14 heures. Chaque spectre est enregistré et sauvegardé. La connaissance de l'énergie des pics d'absorption totale permet, grâce à une bibliothèque de radio-isotopes fournie avec le logiciel d'acquisition, de retrouver la nature du radio-isotope présent dans l'échantillon. 
TABLEAU I

Constantes physiques relatives aux radio-isotopes observés. Physical radio-isotopes constants.

\begin{tabular}{lccccc}
\hline & ${ }^{212} \mathrm{~Pb}$ & ${ }^{235} \mathrm{U}$ & ${ }^{226} \mathrm{Ra}$ & ${ }^{214} \mathrm{~Pb}$ & ${ }^{214} \mathrm{Bi}$ \\
\hline Famille & ${ }^{232} \mathrm{Th}$ & ${ }^{235} \mathrm{U}$ & ${ }^{238} \mathrm{U}$ & ${ }^{238} \mathrm{U}$ & ${ }^{238} \mathrm{U}$ \\
Période & $10,64 \mathrm{~h}$ & $0.7 \times 10^{9}$ ans & 1600 ans & $26,8 \mathrm{~min}$ & 19,7 min \\
\hline Raie gamma $(\mathrm{keV})$ & 77 & 186 & 186 & 352 & 609 \\
Probabilité $P_{\gamma}(\%)$ & 17,7 & 53,0 & 3,28 & 37,1 & 46,1 \\
\hline
\end{tabular}

La fourniture du spectre de bruit de fond ainsi que de la courbe d'efficacité au logiciel lui permet de calculer automatiquement les activités spécifiques partielles en appliquant la formule :

$$
A=\frac{N_{e}-N_{v}}{m t \varepsilon_{\gamma} P_{\gamma}}
$$

avec $A$ : activité spécifique du radio-isotope exprimée en $\mathrm{Bq} / \mathrm{kg}, N_{e}$ : nombre de coups sous le pic d'absorption totale du spectre de l'échantillon, $N_{v}$ : nombre de coups sous le pic d'absorption totale du spectre de bruit de fond, $t$ : temps de comptage $(50000 \mathrm{~s}), \varepsilon_{\gamma}$ : efficacité de détection à l'énergie $E_{\gamma}, P_{\gamma}$ : probabilité d'émission de la raie gamma caractéristique, $m$ : masse de l'échantillon en $\mathrm{kg}$.

L'analyse des échantillons a révélé la présence des cinq radio-isotopes : ${ }^{212} \mathrm{~Pb}$, ${ }^{226} \mathrm{Ra},{ }^{214} \mathrm{~Pb},{ }^{214} \mathrm{Bi}$ et ${ }^{235} \mathrm{U}$. Les radioéléments: ${ }^{226} \mathrm{Ra},{ }^{214} \mathrm{~Pb}$ et ${ }^{214} \mathrm{Bi}$, appartiennent tous à la famille de l'uranium-238. Le ${ }^{212} \mathrm{~Pb}$ appartient à la famille du thorium-232. Les constantes physiques utilisées relatives à ces radio-isotopes sont listées dans le tableau I (Browne et Firestone, 1986).

\section{Calcul d'erreurs}

\subsection{Erreurs systématiques}

Seule l'erreur systématique sur la détermination de l'efficacité de détection $\varepsilon_{\gamma}$ est significative ; elle est de l'ordre de 2 à $3 \%$.

Les erreurs systématiques dues à la mesure de la masse de l'échantillon, à celle du temps de comptage et celle relative à la détermination de la probabilité d'émission de la raie gamma concernée ont été négligées.

\subsection{Erreurs statistiques}

Le comptage du bruit de fond est systématiquement soustrait de celui de chacun des pics d'absorption totale. L'erreur engendrée par cette soustraction a été prise 
en compte en rajoutant le comptage à vide à celui des spectres. L'erreur statistique est restée, pour l'ensemble des échantillons, inférieure à un pour-cent.

\subsection{Erreur globale}

L'erreur globale, à deux écarts standards $(2 \sigma)$, entachant le calcul des activités, a été prise égale au double de la somme quadratique des deux erreurs statistique et systématique $\operatorname{sur} \varepsilon_{\gamma}$. Elle est de l'ordre de $5 \%$ pour l'ensemble des mesures d'activités effectuées.

\section{Résultats des mesures}

\subsection{Famille de l'uranium-238}

La raie d'énergie $352 \mathrm{keV}$ utilisée pour le calcul de l'activité partielle du ${ }^{214} \mathrm{~Pb}$ est pure. Il en est de même de la raie d'énergie $609 \mathrm{keV}$ relative au ${ }^{214} \mathrm{Bi}$. Les activités spécifiques de ces deux radio-isotopes sont données, en fonction de l'emplacement de l'échantillon par rapport à l'usine, dans le tableau II.

TABLEAU II

Activités spécifiques expérimentales en Bq/kg. Famille de l'uranium-238. Measured specific activity ${ }^{238} \mathrm{U}$ series.

\begin{tabular}{|c|c|c|c|c|c|c|c|c|c|}
\hline & & \multicolumn{8}{|c|}{$X(\mathbf{m})$} \\
\hline & & \multicolumn{2}{|c|}{0} & \multicolumn{2}{|c|}{300} & \multicolumn{2}{|c|}{800} & \multicolumn{2}{|c|}{1300} \\
\hline & & ${ }^{214} \mathrm{~Pb}$ & ${ }^{214} \mathrm{Bi}$ & ${ }^{214} \mathrm{~Pb}$ & ${ }^{214} \mathrm{Bi}$ & ${ }^{214} \mathrm{~Pb}$ & ${ }^{214} \mathrm{Bi}$ & ${ }^{214} \mathrm{~Pb}$ & ${ }^{214} \mathrm{Bi}$ \\
\hline & -2 & 153 & 150 & 715 & 674 & 432 & 397 & 379 & 350 \\
\hline $\boldsymbol{Y}(\mathbf{m})$ & 2 & 241 & 240 & 635 & 596 & 512 & 473 & 486 & 454 \\
\hline & 7 & 165 & 155 & 874 & 826 & 677 & 631 & 509 & 471 \\
\hline
\end{tabular}

La raie d'énergie $186 \mathrm{keV}$ est mixte et concerne les deux radio-isotopes radium-226 et l'uranium-235. Par conséquent on peut déterminer l'activité partielle du radium-226 par deux méthodes.

La première méthode consiste à supposer que le radium-226 est en équilibre radioactif avec ses premiers descendants solides : ${ }^{214} \mathrm{~Pb}$ et ${ }^{214} \mathrm{Bi}$ et en prenant comme activité partielle du radium-226 la valeur moyenne des activités de ces deux radio-isotopes, qui par ailleurs sont presque identiques puisqu'ils sont en équilibre :

$$
A_{\mathrm{Ra} 226}=\frac{A_{\mathrm{Pb} 214}+A_{\mathrm{Bi} 214}}{2} .
$$

Pour faciliter la compréhension des formules, les notations habituelles des radionucléides n'ont pas été respectées. 
La deuxième méthode consiste à écrire :

$$
C_{186 \mathrm{keV}}=P_{\mathrm{Ra} 226}^{\gamma} A_{\mathrm{Ra} 226}+P_{\mathrm{U} 235}^{\gamma} A_{\mathrm{U} 235}
$$

avec :

$C_{186 \mathrm{kev}}$ : taux de comptage corrigé de l'efficacité et du rendement du détecteur pour la raie $186 \mathrm{keV}$,

$A_{\text {Ra226 }}$ : activité du radium-226 dans l'échantillon,

$A_{\mathrm{U} 235}$ : activité de l'uranium-235 dans l'échantillon,

$P_{\mathrm{R} \text { 226 }}^{\gamma}$ : probabilité d'émission de la raie gamma $186 \mathrm{keV}$ du radium-226,

$P_{\mathrm{U} 235}^{\gamma}$ : probabilité d'émission de la raie gamma $186 \mathrm{keV}$ de l'uranium-235.

En introduisant :

$\lambda_{\mathrm{Ra} 226}=$ constante radioactive du radium-226,

$\lambda_{\mathrm{U} 235}=$ constante radioactive de l'uranium-235,

$N_{\mathrm{U} 235}=$ nombre total de noyaux ${ }^{235} \mathrm{U}$ contenu dans l'échantillon après traitement,

$N_{\text {Ra226 }}=$ nombre total de noyaux ${ }^{226} \mathrm{Ra}$ contenu dans l'échantillon après traitement,

on peut écrire : $C_{186 \mathrm{keV}}=P_{\mathrm{Ra} 226}^{\gamma} \lambda_{\mathrm{Ra} 226} N_{\mathrm{Ra} 226}+P_{\mathrm{U} 235}^{\gamma} \lambda_{\mathrm{U} 235} N_{\mathrm{U} 235}$. Et en faisant intervenir :

$N_{\text {Ra226 }}^{\text {nat }}=$ nombre total de noyaux ${ }^{226} \mathrm{Ra}$ contenu dans l'échantillon naturel,

$N_{\mathrm{U} 238}^{\text {nat }}=$ nombre total de noyaux ${ }^{238} \mathrm{U}$ contenu dans l'échantillon naturel,

on aura :

$$
C_{186 \mathrm{keV}}=P_{\mathrm{Ra} 226}^{\gamma} \lambda_{\mathrm{Ra} 226} N_{\mathrm{Ra} 226}^{\text {nat }} \frac{N_{\mathrm{Ra} 226}}{N_{\mathrm{Ra} 226}^{\text {nat }}}+P_{\mathrm{U} 235}^{\gamma} \frac{\lambda_{\mathrm{U} 235}}{\lambda_{\mathrm{U} 238}} \lambda_{\mathrm{U} 238} N_{\mathrm{U} 238}^{\text {nat }} \frac{N_{\mathrm{U} 238}}{N_{\mathrm{U} 238}^{\text {nat }}} \frac{N_{\mathrm{U} 235}}{N_{\mathrm{U} 238}} .
$$

Soient $x_{\mathrm{Ra} 226}=\frac{N_{\mathrm{Ra} 226}}{N_{\mathrm{Ra} 226}^{\text {nat }}}$ et $x_{\mathrm{U} 238}=\frac{N_{\mathrm{U} 238}}{N_{\mathrm{U} 238}^{\text {nat }}}$ les résidus respectivement $\mathrm{du}$ radium-226 et de l'uranium-238 dans les échantillons. Alors, on peut écrire :

$$
C_{186 \mathrm{keV}}=P_{\mathrm{Ra} 226}^{\gamma} A_{\mathrm{Ra} 226}^{\mathrm{nat}} x_{\mathrm{Ra} 226}+P_{\mathrm{U} 235}^{\gamma} \frac{\lambda_{\mathrm{U} 235}}{\lambda_{\mathrm{U} 238}} A_{\mathrm{U} 238}^{\mathrm{nat}} x_{\mathrm{U} 238} \frac{N_{\mathrm{U} 235}}{N_{\mathrm{U} 238}}
$$

où $A_{\mathrm{Ra} 26}^{\text {nat }}=\lambda_{\mathrm{Ra} 226} N_{\mathrm{Ra} 226}^{\text {nat }}$ et $A_{\mathrm{U} 238}^{\text {nat }}=\lambda_{\mathrm{Ra} 226} N_{\mathrm{U} 238}^{\text {nat }}$ sont les activités respectivement du radium-226 et de l'uranium-238 dans un minerai de phosphate naturel. 
En admettant que :

$$
A_{\mathrm{Ra} 226}^{\mathrm{nat}}=A_{\mathrm{U} 238}^{\mathrm{nat}} \text { (équilibre séculaire) }
$$

on peut écrire :

$$
C_{186 \mathrm{keV}}=\left(1+\frac{P_{\mathrm{U} 235}^{\gamma}}{P_{\mathrm{Ra} 226}^{\gamma}} \frac{\lambda_{\mathrm{U} 235}}{\lambda_{\mathrm{U} 238}} \frac{x_{\mathrm{U} 238}}{x_{\mathrm{Ra} 226}} \frac{N_{\mathrm{U} 235}}{N_{\mathrm{U} 238}}\right) P_{\mathrm{Ra} 226}^{\gamma} A_{\mathrm{Ra} 226}^{\mathrm{na1}} x_{\mathrm{Ra} 26} .
$$

Ce qui montre que :

$$
x_{\mathrm{Ra} 226} A_{\mathrm{Ra} 226}^{\mathrm{nat}}=\frac{1}{\left(1+\frac{P_{\mathrm{U} 235}^{\gamma}}{P_{\mathrm{Ra} 226}^{\gamma}} \frac{\lambda_{\mathrm{U} 235}}{\lambda_{\mathrm{U} 238}} \frac{x_{\mathrm{U} 238}}{x_{\mathrm{Ra} 226}} \frac{N_{\mathrm{U} 235}}{N_{\mathrm{U} 238}}\right)} \frac{C_{\text {186keV }}}{P_{\mathrm{Ra} 226}^{\gamma}} .
$$

Cette expression s'écrit sous la forme :

$$
A_{\mathrm{Ra} 226}=k_{\mathrm{Ra} 26} A_{\mathrm{Ra} 226}^{\prime}
$$

où

$A_{\mathrm{Ra} 226}=x_{\mathrm{Ra} 226} A_{\mathrm{Ra} 226}^{\mathrm{nat}}=\lambda_{\mathrm{Ra} 226} N_{\mathrm{Ra} 226}$ qui est l'activité du radium-226 dans l'échantillon,

$A_{\mathrm{Ra} 26}^{\prime}=\frac{C_{186 \mathrm{keV}}}{P_{\mathrm{Ra} 26}^{\gamma}}$ représente l'activité du radium-226 dans l'échantillon en

l'absence d'uranium-235,

$$
k_{\mathrm{Ra} 226}=\frac{1}{\left(1+\frac{P_{\mathrm{U} 235}^{\gamma}}{P_{\mathrm{R} 2226}^{\gamma}} \frac{\lambda_{\mathrm{U} 235}}{\lambda_{\mathrm{U} 238}} \frac{x_{\mathrm{U} 238}}{x_{\mathrm{Ra} 226}} \frac{N_{\mathrm{U} 235}}{N_{\mathrm{U} 238}}\right)} .
$$

En introduisant les abondances isotopiques $a_{\mathrm{U} 235}$ et $a_{\mathrm{U} 238}$ respectivement de l'uranium-235 et de l'uranium-238 et leurs périodes respectives $T_{\mathrm{U} 235}$ et $T_{\mathrm{U} 238}$ on peut écrire : $\operatorname{car} \frac{N_{\mathrm{U} 235}}{N_{\mathrm{U} 238}}=\frac{238}{235} \frac{a_{\mathrm{U} 235}}{a_{\mathrm{U} 238}}$

$$
k_{\mathrm{Ra} 226}=\frac{1}{\left(1+\frac{238}{235} \frac{\left.P_{\mathrm{U} 235}^{\gamma} \frac{T_{\mathrm{U} 238}}{P_{\mathrm{R} 1226}^{\gamma}} \frac{x_{\mathrm{U} 238}}{T_{\mathrm{U} 235}} \frac{a_{\mathrm{U} 235}}{x_{\mathrm{Ra} 226}}\right)}{a_{\mathrm{U} 238}}\right)}
$$


En prenant comme abondances isotopiques $a_{\mathrm{U} 235}=0,72 \%$ et $a_{\mathrm{U} 238}=99,28 \%$ et en utilisant les valeurs des résidus dans le phosphogypse $x_{\mathrm{Ra} 226}=80 \%$ et $a_{\mathrm{U} 238}=15 \%$ (USEPA, 1993) ce qui correspond à un rapport des résidus $\frac{x_{\mathrm{Ra} 266}}{x_{\mathrm{U} 238}}=5,33$ on trouve un coefficient $k_{\mathrm{Ra} 226}=0,876$ et les activités du radium226 sont présentées dans le tableau VII.

On constate qu'il y a un écart assez important entre les valeurs de l'activité du radium-226 déduites à partir des formules (2) et (3). Ce qui prouve que nos échantillons ne sont pas formés uniquement de phosphogypse. Cependant si on utilise un rapport des résidus $\frac{x_{\mathrm{Ra} 226}}{x_{\mathrm{U} 238}}=1,3$ (Silva et al., 2001); on trouve $k_{\mathrm{Ra} 226}=0,632$ et on constate que les valeurs de l'activité du radium-226 déduites à partir des formules (2) et (3) sont presque identiques (Tab. VII).

\subsection{Famille de l'uranium-235}

L'application d'une formule similaire à (3) permet de calculer l'activité spécifique due à l'uranium-235, elle s'écrit :

$$
A_{\mathrm{U} 235}=k_{\mathrm{U} 235^{A}}^{\prime}{ }_{\mathrm{U} 235}
$$

où $A_{\mathrm{U} 235}^{\prime}$ serait l'activité de l'uranium-235 en l'absence totale du radium-226 et

$$
k_{\mathrm{U} 235}=\frac{1}{\left(1+\frac{235}{238} \frac{P_{\mathrm{Ra} 226}^{\gamma}}{P_{\mathrm{U} 235}^{\gamma}} \frac{T_{\mathrm{U} 235}}{T_{\mathrm{U} 238}} \frac{x_{\mathrm{Ra} 226}}{x_{\mathrm{U} 238}} \frac{a_{\mathrm{U} 238}}{a_{\mathrm{U} 235}}\right)} .
$$

En utilisant les mêmes constantes physiques que dans le cas du rapport des résidus $\frac{x_{\mathrm{Ra} 226}}{x_{\mathrm{U} 238}}=1,3$, on trouve un coefficient $k_{\mathrm{U} 235}=0,368$. La somme des deux coefficients $k$ est bien égale à 1 .

L'activité spécifique relative à l'uranium-235 calculée ainsi est listée, en fonction de l'emplacement de l'échantillon, dans le tableau III.

\subsection{Famille du thorium-232}

L'activité spécifique du ${ }^{212} \mathrm{~Pb}$, le seul radio-isotope observé de la famille $\mathrm{du}^{232} \mathrm{Th}$, est donnée dans le tableau IV. 
TABLEAU III

Activités spécifiques en Bq/kg de l'uranium-235. Specific activity ${ }^{235} \mathrm{U}$ series.

\begin{tabular}{cccccc}
\hline & & \multicolumn{3}{c}{$\boldsymbol{X}(\mathbf{m})$} & \\
\cline { 2 - 5 } & & $\mathbf{0}$ & $\mathbf{3 0 0}$ & $\mathbf{8 0 0}$ & $\mathbf{1 3 0 0}$ \\
\hline $\boldsymbol{Y}(\mathbf{m})$ & $\mathbf{2}$ & 4 & 23 & 16 & 13 \\
& $\mathbf{2}$ & $\mathbf{9}$ & 21 & 19 & 18 \\
& $\mathbf{7}$ & 6 & 28 & 21 & 17 \\
\hline
\end{tabular}

TABLEAU IV

Activités spécifiques en $\mathrm{Bq} / \mathrm{kg}$ du ${ }^{212} \mathrm{~Pb}$. Famille du thorium-232. Specific activity ${ }^{232}$ Th series.

\begin{tabular}{|c|c|c|c|c|c|}
\hline & & \multicolumn{4}{|c|}{$X(\mathrm{~m})$} \\
\hline & & 0 & 300 & 800 & 1300 \\
\hline & -2 & 53 & 143 & 92 & 97 \\
\hline \multirow[t]{2}{*}{$Y(\mathrm{~m})$} & 2 & 91 & 207 & 112 & 113 \\
\hline & 7 & 56 & 219 & 171 & 171 \\
\hline
\end{tabular}

\section{TABLEAU V}

Activités spécifiques gamma en $\mathrm{Bq} / \mathrm{kg}$ (valeurs moyennes) en fonction de la distance par rapport à l'usine.

Gamma specific activity in $\mathrm{Bq} / \mathrm{kg}$ (mean values) as a function of the distance to the plant.

\begin{tabular}{|cccc}
\hline \multicolumn{5}{c}{$X(\mathrm{~m})$} & & \\
\hline 0 & 300 & $\mathbf{8 0 0}$ & $\mathbf{1 3 0 0}$ \\
\hline $600 \pm 30$ & $2100 \pm 105$ & $1675 \pm 85$ & $1435 \pm 70$ \\
\hline
\end{tabular}

\section{Interprétation des résultats}

\subsection{Activité spécifique gamma en fonction de la distance par rapport à l'usine}

La valeur moyenne de l'activité spécifique gamma en fonction de la distance par rapport à l'usine est indiquée dans le tableau V.

Loin de la bouche d'évacuation (à partir de 300 mètres) nous constatons que l'activité spécifique moyenne mesurée est égale à $1730 \mathrm{~Bq} / \mathrm{kg}$.

À une distance de plus d'un kilomètre du site l'activité spécifique, de plus de $1400 \mathrm{~Bq} / \mathrm{kg}$, ne diminue que du tiers de la valeur maximale enregistrée à 300 mètres. 


\section{TABLEAU VI}

Activités spécifiques gamma en Bq/kg (valeurs moyennes) en fonction de la distance par rapport à la côte.

Gamma specific activity in $\mathrm{Bq} / \mathrm{kg}$ (mean values) as a function of the distance to the sea shore.

\begin{tabular}{ccc}
\hline & $Y(\mathbf{m})$ & \\
\hline-2 & 2 & 7 \\
\hline $1550 \pm 75$ & $1720 \pm 85$ & $1930 \pm 95$ \\
\hline
\end{tabular}

TABLEAU VII

Activités spécifiques du radium-226 en Bq/kg. ${ }^{226} \mathrm{Ra}$ specific activity in $\mathrm{Bq} / \mathrm{kg}$.

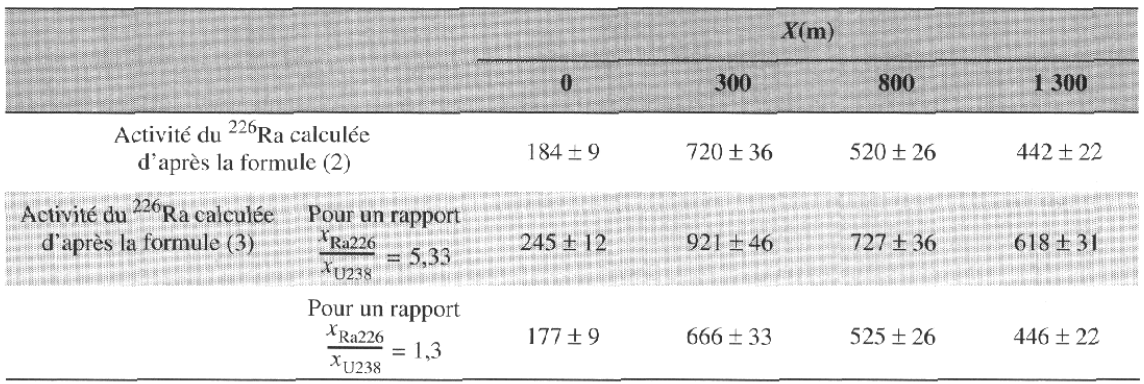

La valeur de l'activité spécifique constatée pour les échantillons prélevés au voisinage de la bouche d'évacuation, relativement faible, est sûrement due au fait que ces échantillons sont composés de boues (déchets de lavage des minerais déversés dans la mer par l'usine) ; contrairement aux autres échantillons qui renferment une quantité importante de poussières de phosphogypse provenant des terrils stockés à l'air libre aux alentours de l'usine (Fig. 1).

\subsection{Activité spécifique gamma en fonction de la distance par rapport à la côte}

L'activité spécifique gamma moyenne, (colonne $x=0 \mathrm{~m}$ exclus), en fonction de la distance par rapport à la côte est indiquée dans le tableau VI.

Sur une bande d'une dizaine de mètres l'activité spécifique reste pratiquement constante, voisine d'une moyenne de $1730 \mathrm{~Bq} / \mathrm{kg}$.

À deux mètres dans l'eau, l'activité gamma spécifique diminue légèrement mais reste toujours importante égale à $1550 \mathrm{~Bq} / \mathrm{kg}$. 


\subsection{Activité partielle spécifique due à la présence du radium-226}

À plus d'un kilomètre du site industriel, l'activité partielle spécifique due à la présence de radium dans le sol est de plus de $400 \mathrm{~Bq} / \mathrm{kg}$ ce qui représente environ le quart de l'activité spécifique gamma.

Mahjoubi et al. (2000) rapportent une activité partielle due au radium-226 de $350 \pm 70 \mathrm{~Bq} / \mathrm{kg}$, valeur moyenne mesurée dans des échantillons prélevés dans des terrils (tabia) de déchets de phosphogypse appartenant à trois usines de traitement dont celle de Skhira.

Selon Charni et al. (1991) la radioactivité partielle due à la présence de radium226, mesurée dans un grand nombre d'échantillons de sols prélevés dans la région de Gafsa et Tozeur en dehors des gisements de phosphates, a une valeur moyenne de l'ordre de $20 \mathrm{~Bq} / \mathrm{kg}$ et qui passe à $80 \mathrm{~Bq} / \mathrm{kg}$ au voisinage des gisements.

\section{Conclusions}

La présence d'une activité gamma de plus de $1700 \mathrm{~Bq} / \mathrm{kg}$ à plus de $1 \mathrm{~km}$ du site de l'usine, due notamment au stockage des phosphogypses à l'air libre engendre donc une pollution de l'environnement aux alentours du site industriel.

\section{RÉFÉRENCES}

Browne E., Fircstone R.B. (1986) Table of radioactive Isotopes. Wiley.

Charni Majoubi H., Abbes A., Aboudi A., Khayati S., Grauby A., Mtimet S. (1991) Étude de la radioactivité naturelle dans le sol du sud tunisien - région de Gafsa Toseur, Radioprotection 26(3), 537-549.

Mahjoubi H., Charfi A.H., Labidi S., Chahed N., Mtimet S. (2000) Estimation des expositions associées à la présence du radon-222 dans les usines de traitement de phosphates et leurs environnements en Tunisie, Radioprotection 35(2), 11-25.

Silva P.S.C., Mazzilli B.P., Favaro D.I.T. (2001) Chemical and Radiological Characterization of Santos Estuary Sediments, VIII Congresso Brasileiro de Geoquimica, Curtiba.

UNSCEAR (1993) Comité Scientifique des Nations Unies pour l'Étude des Effets des Rayonnements Ionisants.-Ioni $\angle$ ing Radiation Sources and Biological Effects. Report To General Assembly, New York.

USEPA (1993) US Environmental Protection Agency, Office of Radiation and Indoor Air, Diffuse NORM Wastes - Waste Characterization and Preliminary Risk Assessment, Draft, RAE9232/1 - 2, SC \& A, Inc., and Rogers \& Associates Engineering Corporation, Salt Lake City, Utah. 